\title{
Uso del Modelo Lineal de un Turboventilador al Análisis de su Funcionamiento con Poscombustión
}

\author{
Oleg A. Khatchatourian y João C.M. Neves \\ Departamento de Física, Estadística y Matemáticas, Universidad de ljuí- UNIJUí, \\ Calle San Francisco, 501. Caja Postal 560, CEP 98700-000. Ijuí, RS-Brasil \\ (e-mail: olegkha@unijui.tche.br)
}

\section{Resumen}

El objetivo de este trabajo fue la comparación de eficacia de sistema de los sostenedores mecánicos y aerodinámicos de llama para el dispositivo de poscombustión de turboventilador. Se propone un modelo para comparar la eficacia de sistemas mecánicos y aerodinámicos de llama y se analiza la influencia de diversos parámetros en la eficacia. El sistema aerodinámico puede ser recomendado como unidad de aumento moderado de empuje para uso en operación de emergencia o en corto tiempo de operación.

Palabras clave: turboventilador, poscombustión, sostenedor aerodinámico, sostenedor mecánico

\section{Application of the Turbofan Linear Model to the Analysis of its Functioning with Afterburner}

\begin{abstract}
The purpose of the presented work was a comparison of an efficiency of Mechanical Flame Holders system and Aerodynamic Flame Holders system for turbofan engine afterburner. The linear mathematical model of the turbofan engine was developed to analyze an influence of air compressor-bleeding for Aerodynamic Flame Holders system on the turbofan engine characteristics. An evaluation criterion was proposed to compare an efficiency of Mechanical and Aerodynamic systems. An influence of differrent parameters on this criterion was analyzed. The factors that cause pressure loss accretion during afterburner down-time, increase the region where the use of Aerodynamic Flame Holders system is preferable in comparison with Mechanical Flame Holders system. It may be concluded that this system can be recommended as moderate thrust augmentation reserve unit for use in emergency operation (insufficient take-off distance, engine failure) or in short- time operation (to break the sound barrier, to reach the most height, take-off).
\end{abstract}

Keywords: turbofan, afterburner, aerodynamic holder, mechanical holder 


\section{INTRODUCCIÓN}

Para mantener el frente de llama en el dispositivo de poscombustión de turborreactores e turboventiladores se utilizan normalmente algunos sostenedores mecánicos (MFH) de la llama que tienen una forma aerodinámica desfavorable. Su defecto es su alta resistencia aerodinámica, que conserva en el régimen sin funcionamiento de poscombustión. La pérdida de la presión reduce perceptiblemente la fuerza propulsiva y el rendimiento económico de motores principalmente en vuelos largos del tiempo en régimen sin forzar. El uso de los sostenedores aerodinámicos (AFH) de la llama, por ejemplo, en la forma de los chorros transversales (Koopman, 1990; Khatchatourian, 1978, 1997 y 1998; Gollahalli y Pardiwalla, 2002, Li et al., 2006) permite que evitemos este problema, porque las regiones de la circulación en el régimen sin forzar están ausentes. Sin embargo, el desvío del aire detrás del compresor al dispositivo de poscombustión perturba el equilibrio de la energía en el motor y causa las alteraciones de sus características. En el actual trabajo estudiamos los cambios de los parámetros de un motor de turboventilador con un compresor de doble carrete en el caso de la utilización de AFH o de MFH en el dispositivo de poscombustión después de mezclarse de flujos. Una comparación económica de estos dos métodos de estabilización durante el tiempo de vuelo entero se realiza.

\section{MODELO LINEAL DE TURBOVENTILADOR}

Los procesos varios y complejos que ocurren en un motor de turboventilador son descritos por el sistema de ecuaciones no lineales, que es difícil de solucionar. Cualquier alteración de un parámetro del turboventilador causa una alteración prácticamente del resto de los pará- metros del turboventilador. Fue mostrado en (Cherkez, 1975; Shliakhtenko, 1985; Urban y Volponi, 1992; Doel, 1994; Simani et al., 2000; Liu y Sirigano, 2001; Balas, 2002; Henrion et al., 2004) que para los motores de la configuración complicada el análisis cuantitativo de relaciones entre los parámetros de los elementos y los parámetros del motor entero se puede realizar con una precisión razonable por la linearización de las ecuaciones no lineales en un intervalo de las desviaciones pequeñas del punto de diseño. Igualando los incrementos de las funciones que describen el proceso del trabajo de motor a sus diferenciales, se obtiene un sistema de las ecuaciones algebraicas lineales para las desviaciones pequeñas de los parámetros del turboventilador, en otras palabras se obtiene un modelo lineal del motor de turboventilador. Usando las recomendaciones de (Cherkez, 1975; Shliakhtenko, 1985), fue obtenido un sistema de las desviaciones pequeñas para un motor de turboventilador con compresor de dos carretes del conducto principal y un dispositivo de poscombustión con un mezclador de los flujos (Fig.1). Este sistema permite que calculemos una influencia de los factores siguientes en los parámetros de turboventilador: la sangría del aire del compresor de alta presión; bypass del aire en dispositivo de poscombustión y la perdída de la presión por resistencia aerodinámica del sostenedor de la llama y por la fuente del calor.

Se asume eso:

- el flujo es uno dimensional en todas las secciones;

- la pérdida del gas y la pérdida de calor del turboventilador a sus alrededores se descuidan;

- el cociente específico del calor para el aire es $\mathrm{k}=1.4$ y para los productos de la combustión es $k=1,33$;

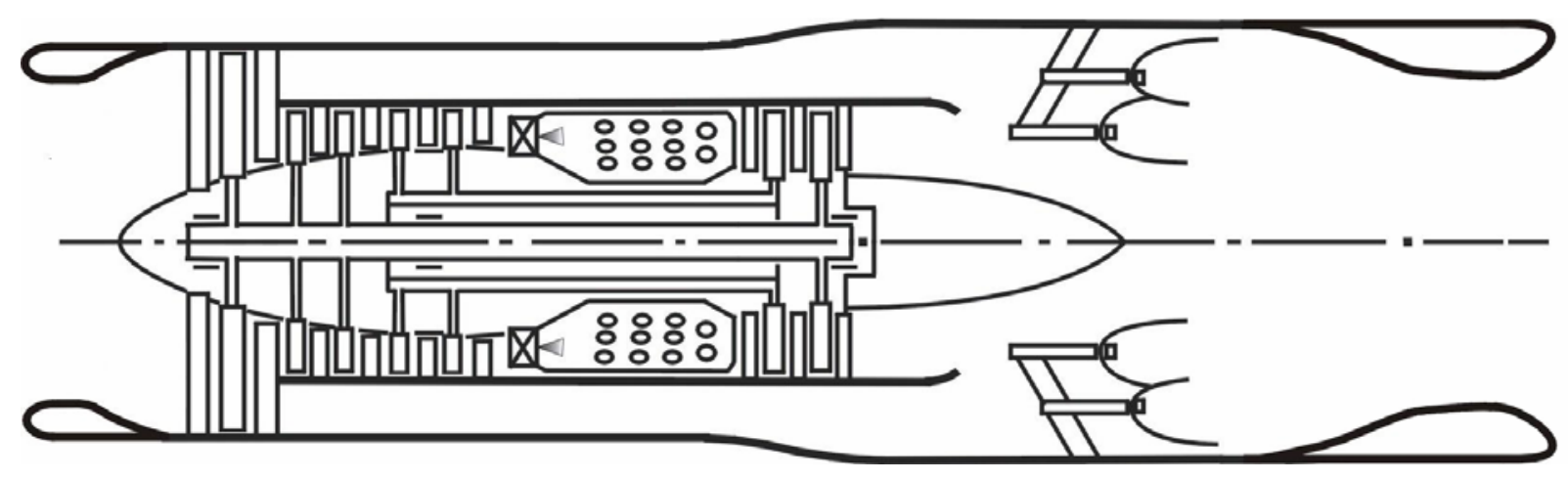

Fig. 1: Bosquejo del motor de turboventilador estudiado. 
- las variaciones del coeficiente de la presión dinámica, del rendimiento, de tamaños geométricos del conducto del flujo del gas-aire de turboventilador (excepto la tobera de escape) y el trabajo de bypass del aire se descuidan.

Usando las mismas notaciones que adentro (Shliakhtenko, 1985), el sistema de la ecuación en las desviaciones pequeñas que describen el proceso de trabajo del motor de turboventilador se puede escribir como:

1. Dependencia entre las relaciones de las presiones:

a) para el conducto principal

$$
\delta \pi_{K 1}+\delta \pi_{K}^{\prime}-\delta \pi_{T 1}-\delta \pi_{T 2}-\delta P_{C 1}=0
$$

b) para el conducto de ventilador

$$
\delta \pi_{K 2}-\delta P_{C 2}=0
$$

2. Ecuaciones de la continuidad:

a) entre la entrada del ventilador y la entrada del mezclador del flujo de aire en el conducto de ventilador

$$
\begin{aligned}
& Q_{m} \delta n_{1}-\left(1-Q_{10}\right) \delta \pi_{K 2}+0.5 \delta T_{K 2}- \\
& Q_{6} \delta \pi_{C 2}=0
\end{aligned}
$$

b) entre la entrada del compresor del conducto principal y los primeros álabes de guía del inyector de la primera turbina

$$
\begin{aligned}
& A_{m} \delta n_{1}-\left(1-A_{10}\right) \delta \pi_{K 1}+0.5 \delta T_{q}-\delta \pi_{K}^{\prime}= \\
& \delta q_{1}+\delta q_{2}+\delta q_{3}
\end{aligned}
$$

c) entre la entrada del primer compresor y la entrada del segundo compresor en conducto principal

$A_{m} \delta n_{1}-\left(1-A_{10}\right) \delta \pi_{K 1}+0.5\left(1+B_{m}\right) \delta T_{K 1}-$

$B_{m} \delta n_{2}-B_{10} \delta \pi_{K 1}=0$

d) entre los álabes de guía del inyector del primer y segundo carrete de la turbina

$\left(1-0.5 A_{3} A_{4}\right) \delta \pi_{T 1}=-\mu_{1} \delta q_{1}$

e) entre los álabes de guía del inyector de la turbina del segundo carrete y la entrada del mezclador del flujo de aire en conducto principal

$\left(1-0.5 B_{3} B_{4}\right) \delta \pi_{T 2}-K_{6} \delta \pi_{C 2}=0$

3. Ecuaciones de la variación de la temperatura:

a) en la pieza del ventilador del conducto de ventilador

$-Q_{2} Q_{n} \delta n_{1}+\delta T_{K 2}-Q_{2} Q_{n} \delta \pi_{K 2}=0$

b) a lo largo del conducto de ventilador

$$
\delta T_{K 2}-\delta T_{C 2}=0
$$

c) en el compresor del conducto principal

$$
-A_{2} A_{n} \delta n_{1}+\delta T_{K 1}-A_{2} A_{1} \delta \pi_{K 1}=0
$$

d) en el carrete de la turbina de alta presión

$$
\delta T_{T 1}-\delta T_{q}+A_{3} A_{4} \delta \pi_{T 1}=0
$$

e) en el carrete de la turbina de baja presión

$$
\delta T_{C 1}-\delta T_{T 1}+B_{3} B_{4} \delta \pi_{T 2}=0
$$

4. Ecuaciones del balance de la energía:

a) en el carrete de alta presión

$$
\begin{aligned}
& B_{n} \delta n_{2}+\left(1-0.5 B_{n}\right) \delta T_{K 1}-A_{3} \delta \pi_{T 1}-\delta T_{q}+ \\
& B_{q} \delta \pi_{K}^{\prime}=N_{1} \delta q_{1}+\delta q_{2}+\delta q_{3}
\end{aligned}
$$

b) en el carrete de la baja presión

$$
\begin{aligned}
& {\left[\left(1-K_{19}\right) A_{n}+K_{19}\left(Q_{m}+Q_{n}-A_{m}\right)\right] \delta n_{1}-} \\
& \delta T_{T 1}-\left[\left(1-K_{19}\right) A_{1}-K_{19} A_{10}\right] \delta \pi_{K 1}+\left(Q_{1}+Q_{10}\right) \\
& K_{19} \delta \pi_{K 2}-B_{3} \delta \pi_{T 2}=-\delta q_{2}-N \delta q_{3}
\end{aligned}
$$

5. Relaciones entre los parámetros del flujo en mezclador del flujo de aire:

a) conducto principal del flujo del gas-aire

$$
\delta \pi_{C 1}-\delta P_{C 1}+\delta P^{\prime}=0
$$

b) conducto de ventilador:

$$
\delta \pi_{C 2}-\delta P_{C 2}+\delta P^{\prime}=0
$$


c) la presión del estancamiento en la salida del mezclador del poscombustión

$$
K_{13} \delta \pi_{C 1}+\left(1-K_{13}\right) \delta P_{C 2}-\delta P_{C}=\delta \sigma_{C S}
$$

d) la temperatura del estancamiento del gas que tiene en cuenta la liberación de calor en el dispositivo de poscombustión

$$
\begin{aligned}
& \left(1-K_{15}\right) \delta T_{C 1}+K_{15} \delta T_{C 2}+\left(K_{16}-K_{15}\right)\left(\delta G_{B 1}\right. \\
& \left.-\delta G_{B 2}\right)-\delta T_{C}=\left(K_{16}-K_{15}\right) \delta q_{2}-\delta q_{3}
\end{aligned}
$$

e) la ecuación del caudal en la salida de la tobera de escape

$$
\begin{aligned}
& \left(1-K_{16}\right) \delta G_{B 1}+K_{16} \delta G_{B 2}-\left(1+K_{6}\right) \delta P_{C}+ \\
& 0.5 \delta T_{C}-\delta F_{C}=\left(1-K_{16}\right) \delta q_{2}
\end{aligned}
$$

6. Variación de la fuerza propulsiva:

$$
\begin{aligned}
& \left(1-K_{16}\right) \delta G_{B 1}+K_{16} \delta G_{B 2}+0.5 K_{3} K_{9} \delta P_{C}- \\
& \delta R_{C}+0.5 K_{9} \delta T_{C}=\left(1-K_{16}\right) \delta q_{2}
\end{aligned}
$$

7. Variación de la consumición de combustible total:

$$
\delta G_{T}-K_{21} \delta G_{T 1}-\left(1-K_{21}\right) \delta G_{T C S}=0
$$

8. Variación de la consumición de combustible en el dispositivo de poscombustión:

$$
\begin{aligned}
& \delta G_{T C S}-\left(1-K_{15}\right) \delta T_{C 1}-K_{15} \delta T_{C 2}-\left(1-K_{15}\right) \\
& \delta G_{B 1}-K_{15} \delta G_{B 2}=K_{5} \delta \theta-\left(1-K_{15}\right) \delta q_{2}
\end{aligned}
$$

9. Variación de la consumición de combustible específica total:

$$
\delta C_{R}-\delta G_{T}+\delta R=0
$$

10. Ecuaciones del equilibrio en el punto del régimen nominal para la curva del funcionamiento del compresor

a) conducto de ventilador

$$
\delta G_{B 1}-A_{m} \delta n_{1}-A_{10} \delta \pi_{K 1}=0
$$

b) conducto principal

$$
\delta G_{B 2}-Q_{m} \delta n_{1}-Q_{10} \delta \pi_{K 2}=0
$$

11. Ecuación de la calefacción del gas en cámara primaria de combustión:

$$
\begin{aligned}
& \delta G_{T 1}-\delta G_{B 1}-K_{5} \delta T_{T}+\left(K_{5}-1\right) \delta T_{K 1}= \\
& -\left(\delta q_{1}+\delta q_{2}+\delta q_{3}\right)
\end{aligned}
$$

donde

$\delta X=\Delta X / X=\left(X_{1}-X_{0}\right) / X_{0}$ es una desviación relativa del valor $X$;

$\delta \pi_{K}\left(\delta \pi_{T}\right)$ es una desviación del relación de la compresión (extensión) en el compresor (turbina);

$\delta P(\delta T)$ es una desviación de la presión (temperatura) del estancamiento;

$\delta G_{B}\left(\delta G_{T}\right)$ es una desviación del caudal másico del aire (consumición de combustible);

$\delta n_{1}\left(\delta n_{2}\right)$ es una desviación de la frecuencia de la rotación del rotor de alta (baja) presión;

$\delta \sigma_{C S}$ es una desviación de la pérdida de la presión de gases en el dispositivo de poscombustión;

$\delta q_{1}$ es una desviación del consumo de aire para refrescarse de álabes de turbina;

$\delta q_{2}$ es una desviación del consumo de aire para uso en interior del avión (sin volverse al motor);

$\delta q_{3}$ es una desviación del consumo de aire para la estabilización aerodinámica en el dispositivo de poscombustión.

Los coeficientes de influencia de entrada $\left(A_{i}\right.$, $\left.B_{i}, Q_{i}, K_{i}\right)$ son calculados por fórmulas dadas adentro (Shliakhtenko, 1985) para los parámetros sabidos del conducto del flujo del gasaire y las curvas del funcionamiento del compresor. Los valores de los coeficientes $A, B$ e $Q$ dependen de parámetros de los compresores de baja y alta presión y del ventilador. Los valores de los coeficientes $K_{i}$ se relacionan con los parámetros del inyector de la cámara primaria de combustión, del dispositivo de poscombustión y de la tobera de escape. Adoptando ciertos valores para $\delta q_{1}, \delta q_{2}, \delta q_{3}$ y $\delta \sigma_{C S}$ y solucionando el sistema de ecuacio- 
nes algebraicas lineales, las desviaciones de los parámetros del turboventilador de sus valores iniciales pueden ser obtenidas. El sistema obtenido de ecuaciones lineales de la orden 26 tiene 29 desconocidas:

$$
\begin{aligned}
& \begin{array}{lllllll}
\delta \pi_{K 1} & \delta \pi_{K 2} & \delta \pi_{K}^{\prime} & \delta \pi_{T 1} & \delta \pi_{T 2} & \delta \pi_{C 1} & \delta \pi_{C 2}
\end{array} \\
& \delta P_{C} \quad \delta P_{C 1} \quad \delta P_{C 2} \quad \delta P^{\prime} \\
& \delta T_{K 1} \delta T_{K 2} \delta T_{C} \quad \delta T_{T 1} \delta T_{g} \delta T_{C 1} \delta T_{C 2} \delta \theta \\
& \delta n_{1} \quad \delta n_{2} \\
& \delta G_{B 1} \quad \delta G_{B 2} \quad \delta G_{T 1} \quad \delta G_{T C S} \quad \delta G_{T} \\
& \delta R \quad \delta C_{R} \quad \delta F_{C}
\end{aligned}
$$

Si se asumir un cierto procedimiento de sistema de regulación, el número las variables desconocidas se puede reducir para formar un sistema determinado de ecuaciones algebraicas lineales. Por ejemplo, en la temperatura constante de ambiente en condiciones nominales de la operación de turboventilador para sistema de regulación con $n_{1}=$ constante, $F=$ constante, es necesario considerar:

$$
\delta F_{C}=0 ; \delta n_{1}=0 ; \delta \theta=0
$$

Para el turboventilador con la calefacción constante en el dispositivo de poscombustión en funcionamiento un sistema de regulación se puede elegir $n_{1}=$ constante, $T_{g}=$ constante, $\mathrm{y}$ es necesario considerar

$\delta T_{g}=0 ; \delta n_{1}=0 ; \delta \theta=0$

La matriz del coeficiente es no singular. El sistema fue solucionado numéricamente por la eliminación gaussiana con la pivotación.

Para verificar la validez del modelo lineal, una comparación entre los resultados numéricos y los datos experimentales sobre la sangría del aire del compresor de alta presión (sin vuelta en conducto del gas-aire) fue realizada bajo condición $n_{1}=$ constante, $F_{C}=$ constante. Los coeficientes experimentales de la influencia fueron obtenidos por el análisis de los datos experimentales de la sangría del aire (el hasta $3 \%$ ) para seis motores de turboventilador. Un acuerdo satisfactorio entre los datos numéricos y experimentales (véase que la tabla 1) demuestra que el modelo matemático corresponde a los procesos físicos.

Los cálculos observados para el motor con el sistema de regulación $n_{1}=$ constante, $T_{g}=$ constante muestran (fig. 2) que bypass del aire en el dispositivo de poscombustión altera a prácticamente todos los parámetros de turboventilador.

Pues el caudal de gas a través del carrete de turbina de presión baja disminuye, para mantener $T_{g}=$ constante es necesario aumentar una diferencia de la presión en la turbina abriendo la tobera de gases. En el mismo tiempo una región de la estabilidad aumenta y una energía requerida para el compresor reduce.

La reducción del caudal en $T_{g}=$ constante en el carrete de alta presión de la turbina resulta a la disminución de la frecuencia de rotación de rotor y a la dislocación del punto de funcionamiento del turbocompresor a la izquierda abajo a lo largo de la curva característica del turbocompresor. Esto causa una reducción del relación de la compresión en el compresor de alta presión y también provoca una disminución pequeña del caudal a través de conducto principal. El carrete de alta presión relacionado aerodinámico con el carrete de baja presión en estas condiciones se puede considerar solamente como cierta "resistencia" que disloque un punto de funcionamiento del compresor de baja presión a la izquierda - para arriba de punto nominal, es decir, ocurre una aproximación pequeña para frontera de bombeo (fig. 3).

Tabla 1: Los coeficientes experimentales y numéricos de la influencia de la sangría.

$$
\left(T_{g}=1203^{\circ} \mathrm{K} ; G_{B 2} / G_{B 1}=1 ; n_{1}=\text { constante; } F_{C}=\text { constante }\right) \text {. }
$$

\begin{tabular}{|c|c|r|r|r|r|r|}
\hline & & $n_{1}$ & $R$ & $G_{T}$ & $C_{R}$ & $T_{q}$ \\
\hline$n_{1}=5300$ & cómputo & 0,13 & $-0,18$ & 1,35 & 1,53 & 1,05 \\
\cline { 2 - 7 } rpm & experimentación & 0,20 & $-0,48$ & 1,21 & 1,69 & 1,26 \\
\hline$n_{1}=5000$ & cómputo & 0,10 & $-0,13$ & 1,42 & 1,55 & 1,02 \\
\cline { 2 - 7 } & expm & 0,19 & -0.48 & 1,32 & 1,8 & 1,77 \\
\hline
\end{tabular}




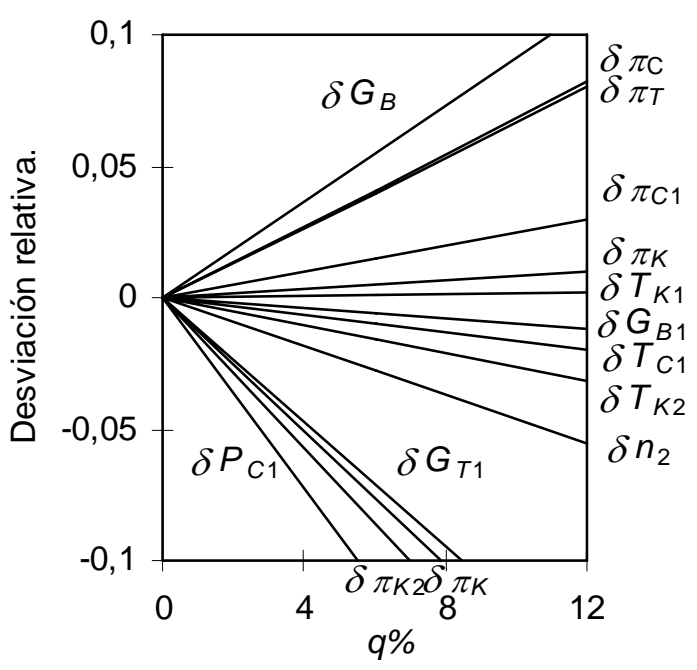

Fig. 2: Variación de los parámetros de turboventilador debido a bypass del aire para la estabilización aerodinámica $(H=0 ; M=0)$.

Es interesante que las desviaciones de la mayoría de parámetros de turboventilador (excepto $R, C_{R}, G_{T . C S}, G_{T}, F_{C}$ ) para un sistema de regulación $n_{1}=$ constante, $T_{g}=$ constante varían lineal cuando el caudal de bypass del aire aumenta. Estas desviaciones no dependen de la resistencia aerodinámica de los sostenedores de la llama, de la perdída de la presión debido a la liberación del calor, del ángulo de la inyección de chorros transversales (sistema de sostenedores aerodinámicos de la llama) y de relación de la calefacción en el poscombustión. En fig. 4 se demuestra las variaciones de la fuerza propulsiva y de la consumición específica de combustible en el dispositivo de poscombustión para $T_{g}=$ constante, $n_{1}=$ constante, $\theta=$ constante.

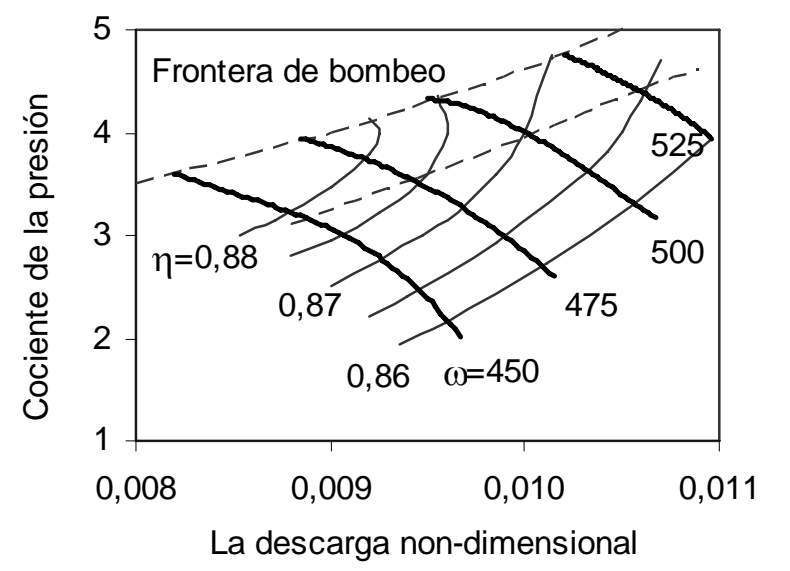

Fig. 3: Curva de funcionamiento de turbocompresor.
La línea discontinua en fig. 4 demuestra una variación de la fuerza propulsiva y de la consumición específica de combustible en función del bloqueo del canal del dispositivo de pos-combustión por MFH (esto es un caso del lí-mite cuando la región de la circulación se crea sin bypass del aire).

El modelo lineal propuesto permite analizar los variaciones de los parámetros del motor para los regímenes transitivos, por ejemplo: (a) en momentos de arranquelparada del dispositivo de poscombustión y bajo variación relativa de la calefacción; (b) debido a un fallo del sistema de abertura de la tobera o a un fallo del sistema de inyección del combustible; (c) cuando es necesario aumentar una fuerza propulsiva en una situación de la emergencia. Estos casos se pueden representar por otros los sistemas de regulación, en detalle, $n_{1}=$ constante, $F_{C}=$ constante.

Las Figuras 5 - 9 demuestran variaciones de los parámetros de turboventilador en diversas secciones del motor para el sistema de regulación $n_{1}=$ constante, $F_{C}=$ constante.

El aumento de la presión ( $P_{C}$ dinámica y $P^{\prime}$ estática) detrás de la turbina (fig. 5) causa una reducción del $\delta \pi_{T 2}$ (fig. 6) que conjuntamente con la reducción del caudal del gas a través de la turbina debido a bypass del aire $\left(\delta G_{B 1} / \delta q<1\right)$, (fig. 8), provoca un aumento de la temperatura del gas antes de la turbina (fig. 7) (para $n_{1}=$ constante).

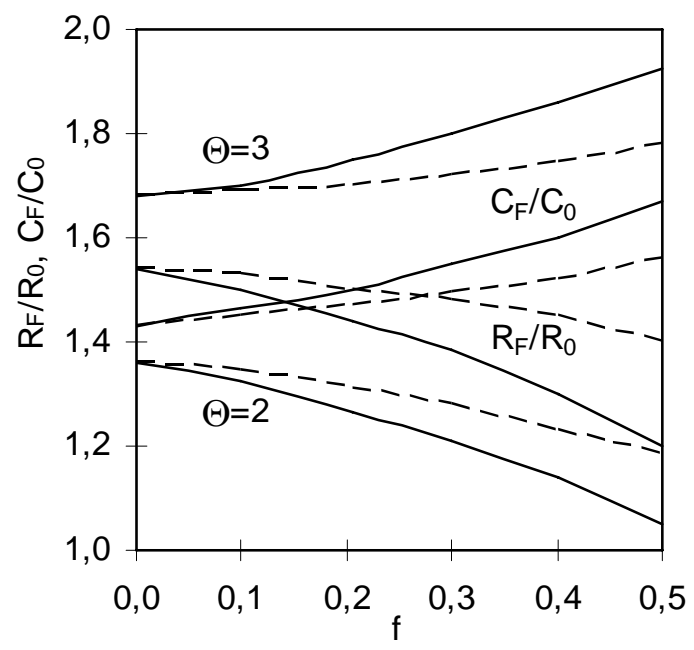

Fig. 4: Influencia de bloqueo del canal y de la calefacción relativa en el dispositivo de poscombustión en la fuerza propulsiva y la consumición de combustible específica $(H=0 ; M=0)$. 


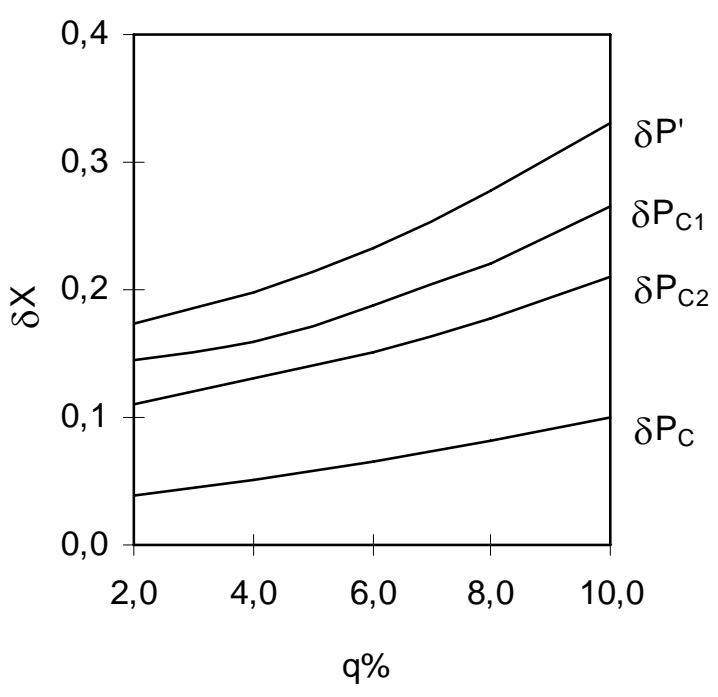

Fig. 5: Variación de presión en diversas secciones de turboventilador debido a bypass del aire para el dispositivo de poscombustión con AFH ( $n_{1}=$ constante, $F_{C}=$ constante).

En el mismo tiempo, el valor $\delta \pi_{K 2}$ del ventilador aumenta (fig. 6), el caudal $G_{B 2}$ a través del conducto principal disminuye (fig. 8) que reduce un margen de la estabilidad del compresor. La subida de la temperatura inmediatamente después de la turbina para el relación de la extensión $\delta \pi_{T 1}=$ constante causa un aumento de la frecuencia $n_{2}$ de la rotación del rotor de alta presión. Por lo tanto, caudal del conducto principal (fig. 8) y la suma de los relaciones de compresiones $\delta \pi_{K}^{\prime}+\delta \pi_{K 1}$ se aumentan (fig. 6).

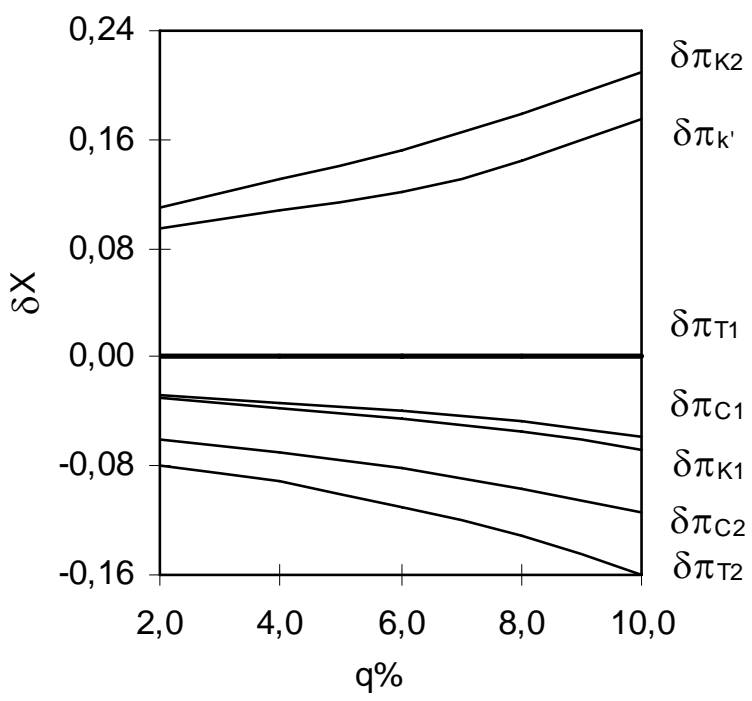

Fig. 6: Variación del relación de la presión en diversas secciones de turboventilador debido a bypass del aire para el dispositivo de poscombustión con AFH ( $n_{1}=$ constante, $F_{C}=$ constante).

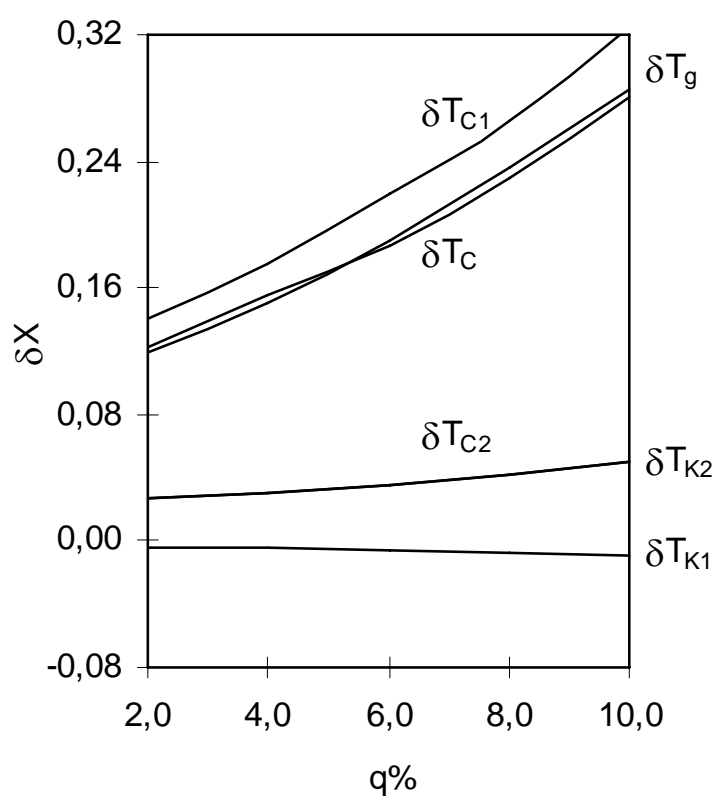

Fig. 7: Variación de la temperatura en diversas secciones de turboventilador debido a bypass del aire para el dispositivo de poscombustión con AFH $\left(n_{1}=\right.$ constante, $F_{C}=$ constante $)$.

Como consecuencia de la subida de la temperatura $T_{C}$ (fig. 7) y del aumento del relación de la presión en la tobera de gases, una fuerza propulsiva aumenta (fig. 9), pero en el mismo tiempo la consumición de combustible específica el $\delta C_{R}$ aumenta (fig. 9), porque las consumiciones de combustible en los compartimientos principales y secundarios aumentan (fig. 8).

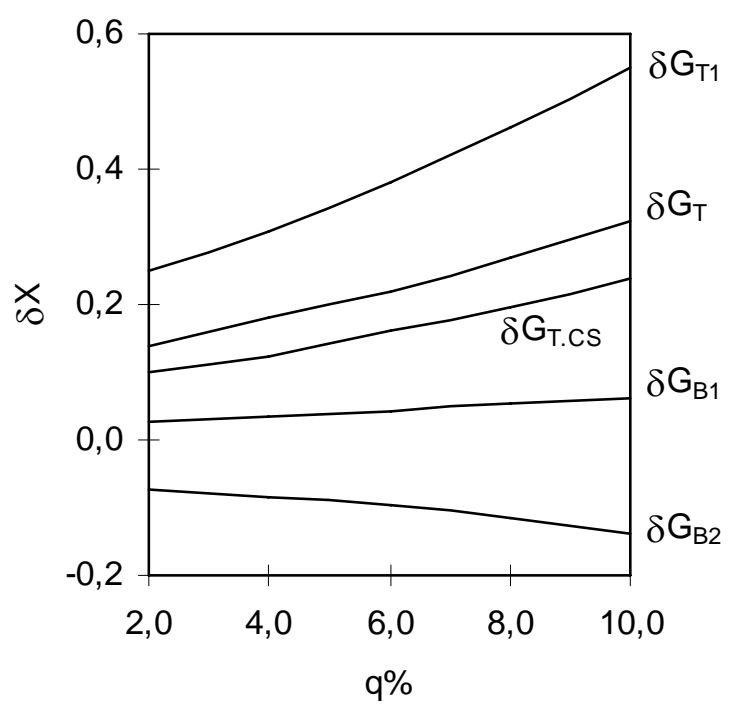

Fig. 8: Variación de los parámetros de turboventilador debido a bypass del aire para el dispositivo de poscombustión con AFH ( $n_{1}=$ constante, $F_{C}=$ constante). 


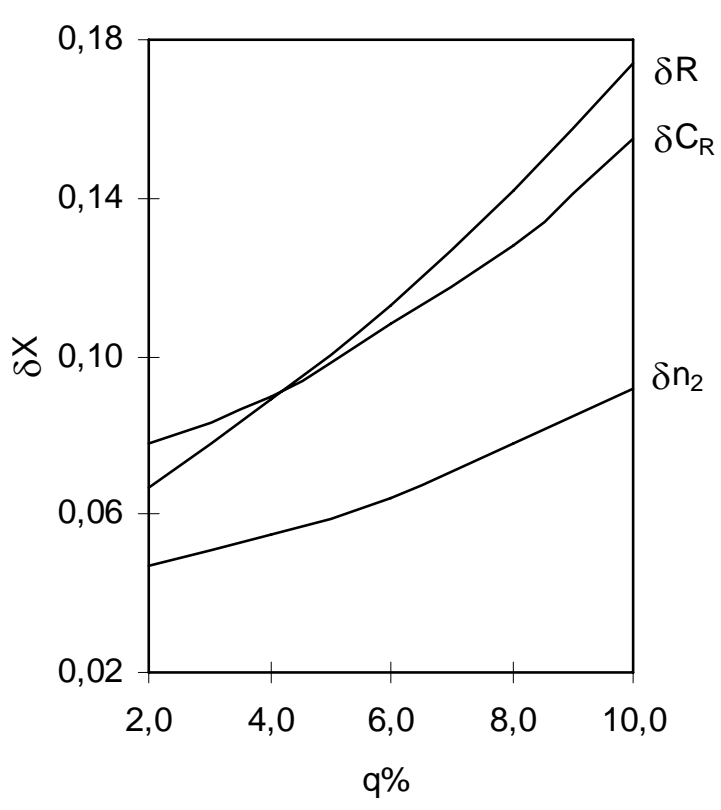

Fig. 9: Variación de los parámetros de turboventila-dor debido a bypass del aire para el dispositivo de poscombustión con AFH $\left(n_{1}=\right.$ constante, $F_{C}=$ constante).

\section{COMPARACIÓN DE SISTEMAS AFH Y MFH}

Usando los resultados del cómputo de interrelaciones entre los parámetros de turboventilador debido a bypass del aire y por caída de la presión en el dispositivo de poscombustión, podemos determinar el sistema más económico de la estabilización por tiempo predeterminado de la operación del dispositivo de poscombustión durante vuelo.

Según (Alverman y Ulken, 1971), el tiempo de vuelo total (tiempo de la operación del motor) $\tau_{\Sigma}$ se divide en dos porciones: 1 ) el ciclo activo del dispositivo de poscombustión (el tiempo de funcionamiento del dispositivo de poscombustión) $\tau_{F}$ y 2) el ciclo inactivo del dispositivo de poscombustión $\left(\tau_{\Sigma}-\tau_{F}\right)$ - el tiempo, durante que el dispositivo de poscombustión no funciona.

La consumición de combustible total durante el vuelo total se puede expresar para los sistemas de MFH y de AFH como:

$M_{T M^{*}} \tau_{\Sigma}=C_{0 M^{*}} \cdot R_{0 M^{*}}\left(\tau_{\Sigma}-\tau_{F}\right)+C_{F M^{*}} R_{F M^{*}} \tau_{F}$

$M_{T A^{\cdot}} \tau_{\Sigma}=C_{0 A^{\cdot}} R_{0 A^{\prime}} \cdot\left(\tau_{\Sigma}-\tau_{F}\right)+C_{F A^{\cdot}} R_{F A^{*}} \tau_{F}$ donde $M_{T}$ es consumición de combustible media durante vuelo total; $C$ es la consumición de combustible específica; $R$ es fuerza propulsiva. Los índices: $0(F)$ - sin (en) el funcionamiento; $M$ - que tiene relación a $\mathrm{MFH} ; A$ - que tiene relación a AFH.

Introduciendo la notación $\tau=\tau_{F} / \tau_{\Sigma}$, podemos obtener $\tau$ para cuál $M_{T A} \leq M_{T M}$ :

$$
\tau \leq \frac{1}{1+\frac{R_{F A} C_{F A}-R_{F M} C_{F M}}{R_{0 M} C_{0 M}-R_{0 A} C_{0 A}}}
$$

Puede ser escrito como:

$$
\begin{aligned}
& \frac{R_{F A}}{R_{F M}}=\frac{R_{F M}+R_{F A}-R_{F M}}{R_{F M}}=1+\delta R_{F A} \cong \\
& \frac{1}{1-\delta R_{F A}}
\end{aligned}
$$

Es análogo

$$
\begin{aligned}
& \frac{R_{0 M}}{R_{0 A}}=1+\delta R_{0 M} \cong \frac{1}{1-\delta R_{0 M}} \\
& \frac{C_{F A}}{C_{F M}}=1+\delta C_{F A} ; \frac{C_{0 M}}{C_{0 A}}=1+\delta C_{0 M} .
\end{aligned}
$$

Es obvio:

$$
\frac{R_{F M}}{R_{0 A}} \frac{C_{F M}}{C_{0 A}}=\frac{R_{F M}}{R_{0 A}} \frac{G_{T 0}+G_{T F}}{G_{T 0}} \frac{R_{0 A}}{R_{F M}}=\frac{1}{K_{21}}
$$

Para realizar una comparación con las fuerzas propulsivas iguales es necesario compensar la reducción de la fuerza propulsiva debido a bypass del aire para sistema de AFH o de perdídas aerodinámicas en caso del sistema de MFH.

Así pues, es necesario aumentar la consumición de combustible en cámara primaria de combustión cuando a) el dispositivo de poscombustión con el sistema de AFH está funcionando, o b) cuando el dispositivo de poscombustión con el sistema de MFH no está funcionando.

Considerando (36) y descuidando el término de la segunda orden, puede ser escrito: 


$$
\begin{aligned}
& \frac{1}{1+\frac{1}{K_{21}} \frac{\left(1+\delta C_{F A}\right)\left(1-\delta R_{F M}\right)-1}{\left(1+\delta C_{0 M}\right)\left(1-\delta R_{0 M}\right)-1}} \cong \\
& \frac{1}{1+\frac{1}{K_{21}} \frac{\delta C_{F A}-\delta R_{F A}}{\delta C_{0 M}-\delta R_{0 M}}}
\end{aligned}
$$

Finalmente

$$
\tau \leq \frac{1}{1+\frac{1}{K_{21}} \frac{\delta C_{F A}-\delta R_{F A}}{\delta C_{0 M}-\delta R_{0 M}}}
$$

Así, sabiendo valores pequeños de la desviación de la consumición de combustible específica y de la fuerza propulsiva debido a bypass del aire para el sistema de AFH, cuando el dispositivo de poscombustión está en una operación y las desviaciones pequeñas debido a las pérdidas de la presión para el sistema de MFH, cuando se cierra el dispositivo de poscombustión, puede encontrar los valores máximos del tiempo de funcionamiento relativo del dispositivo de poscombustión en el tiempo de vuelo total, durante el cual el uso del sistema de AFH es más económico que el uso del sistema de MFH.

Aplicando el modelo elaborado y la desigualdad obtenida (38), una comparación fue hecha entre el dispositivo de poscombustión con sistema aerodinámico de la llama en la forma de los chorros circulares transversales y el sistema (mecánico) de sostenedores de llama en forma de las cuñas con ángulo $60^{\circ}$.

Las superficies demostradas en las figuras 10 - 13 representan los valores de $\tau$, para los cuales las consumiciones de combustible son iguales para ambos sistemas de la estabilización de la llama (con los mismos valores de fuerzas propulsivas y de relacións del bloqueo del canal). Las dimensiones de sostenedores de llama que proveen los mismos límites de la estabilidad fueron determinadas por los fórmulas propuestos por Gilyazov et al. (1975) para el sistema de AFH y Raushenbah el al. (1964) para el sistema de MFH. Se asume que las pérdidas totales de la presión (aerodinámicas y termales) en régimen de la operación del dispositivo de poscombustión son iguales para los sistemas de MFH y de AFH. Las pérdidas aerodinámicas en el dispositivo de poscombustión con los sostenedores de llama en forma de las cuñas con ángulo $60^{\circ}$ eran calculadas por siguiente fórmula (Raushenbah et al., 1964):

$\sigma_{A}=1-\xi \cdot k \cdot M^{2} / 2$

donde: $\xi=(\sqrt{\eta \cdot f}+f)^{2} /(1-f)^{2}$ es el coeficiente de pérdidas aerodinámicas; $\eta$ es el coeficiente de la forma del sostenedor, que depende de geometría del sostenedor. Por ejemplo, para sostenedores de la llama en forma de las cuñas con ángulo $60^{\circ}$ el coeficiente de la forma $\eta$ es igual 0.2.

Para calcular la pérdida de la presión debido a la liberación del calor en el dispositivo de pos-combustión, las ecuaciones sin dimensiones del flujo del gas calentado en un canal cilíndri-co fueron solucionadas:

$\sigma_{T}=q\left(\lambda_{2}\right) / q\left(\lambda_{1}\right) \cdot \sqrt{\theta}$

donde

$q(\lambda)=\left(\frac{k+1}{2}\right)^{\frac{1}{k-1}} \cdot \lambda\left(1-\frac{k-1}{k+1} \lambda^{2}\right)^{\frac{1}{k-1}}$

El coeficiente de la velocidad $\lambda_{2}$ fue obtenido solucionando la ecuación:

$$
Z\left(\lambda_{2}\right)=\lambda_{2}+1 / \lambda_{2}=Z\left(\lambda_{1}\right) / \sqrt{\theta}
$$

La pérdida total de la presión, el caudal necesario de aire para el sistema de AFH y el número de sostenedores necesarios eran calculados por fórmulas siguientes:

$$
\begin{aligned}
& \sigma_{C S}=\sigma_{A} \cdot \sigma_{T} \\
& \Delta G=\mu \cdot n \cdot F_{V} \cdot P_{V} \cdot q\left(\lambda_{V}\right) / \sqrt{T_{V}} \\
& n=F \cdot\left(D_{C S} / D_{Z R}\right)^{2}
\end{aligned}
$$

Las simulaciones indican que el valor de $\tau$ se eleva cuando la calefacción relativa en el dispositivo de poscombustión reduce. En otras palabras, una ventaja del sistema $\mathrm{AFH}$ aumenta cuando requiere un aumento moderado de empuje. 


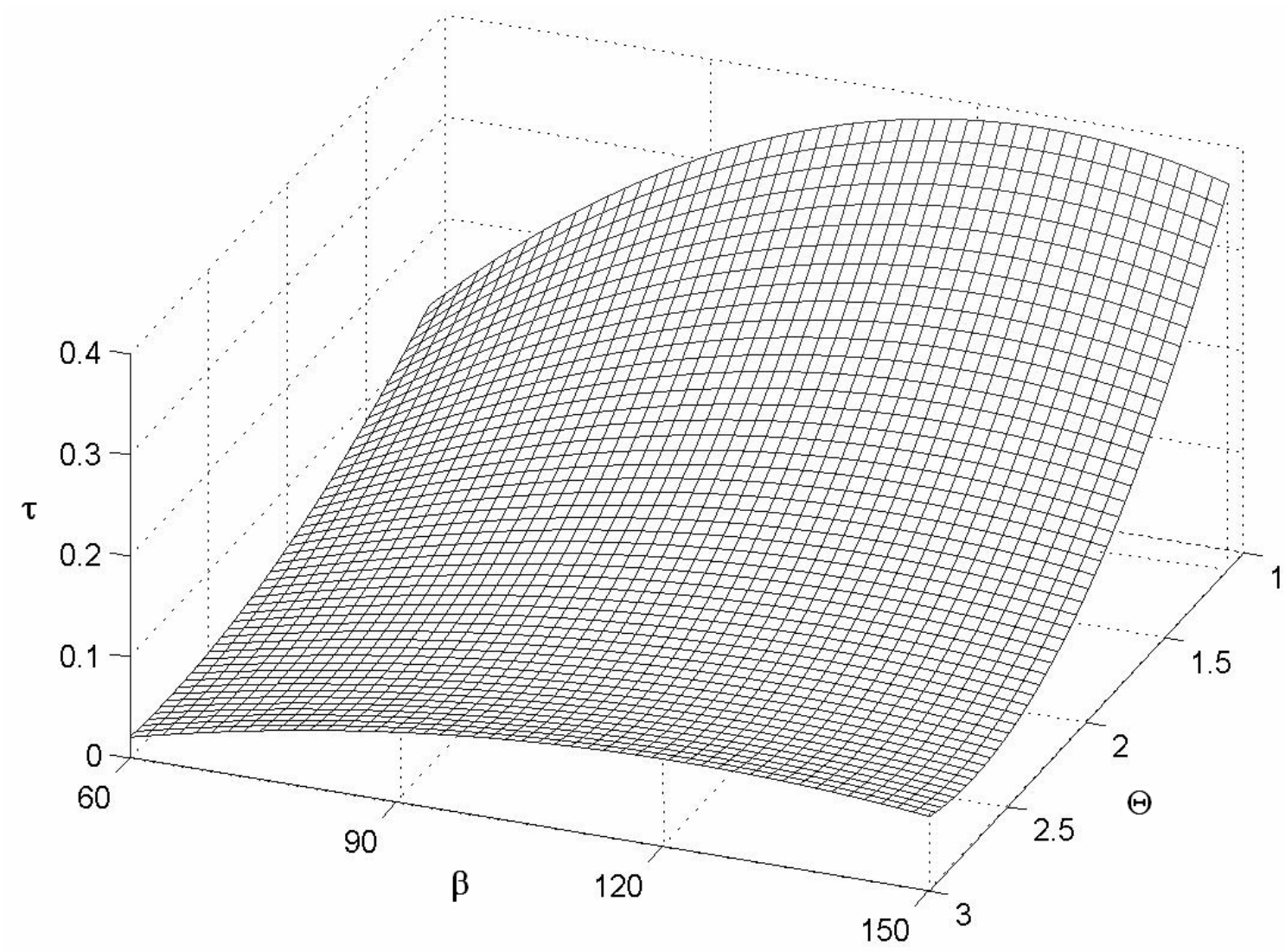

Fig. 10: Influencia del ángulo de la inyección de chorros transversales en valor de $\tau$.

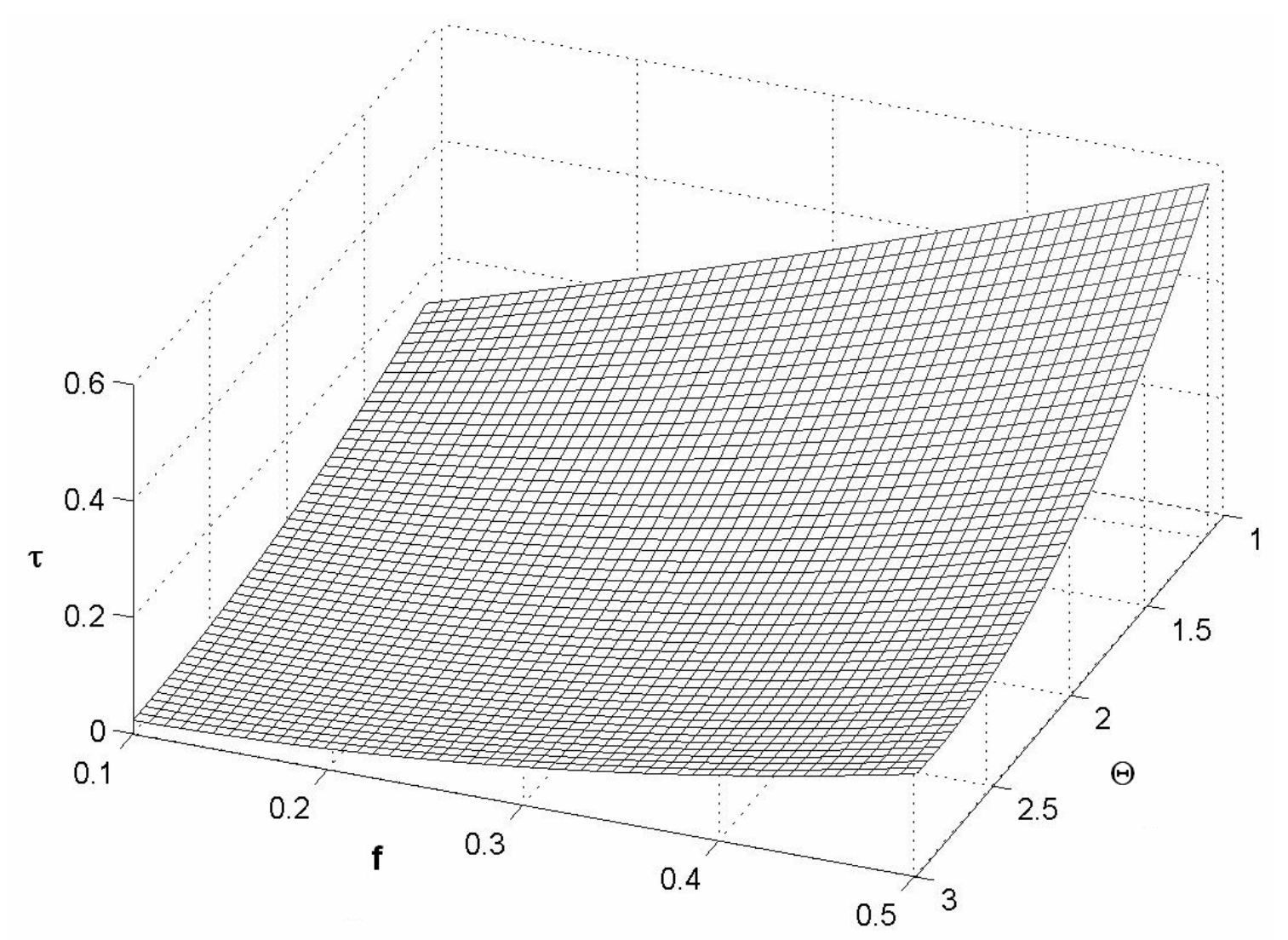

Fig. 11: Influencia de bloqueo del canal y de la calefacción relativa en valor de $\tau$. 


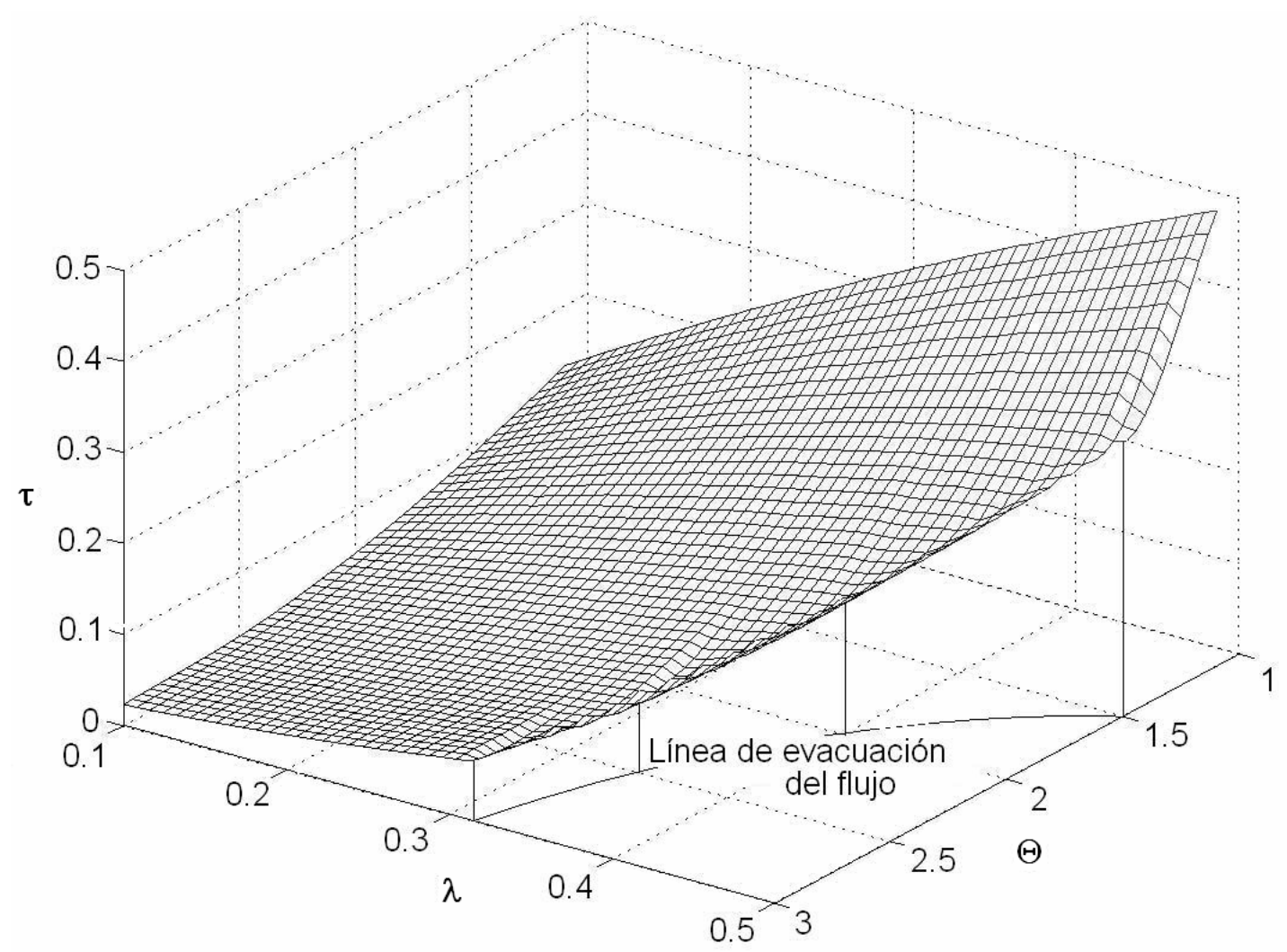

Fig. 12: Influencia de la velocidad de aire en la entrada del dispositivo de poscombustión en el valor de $\tau$.

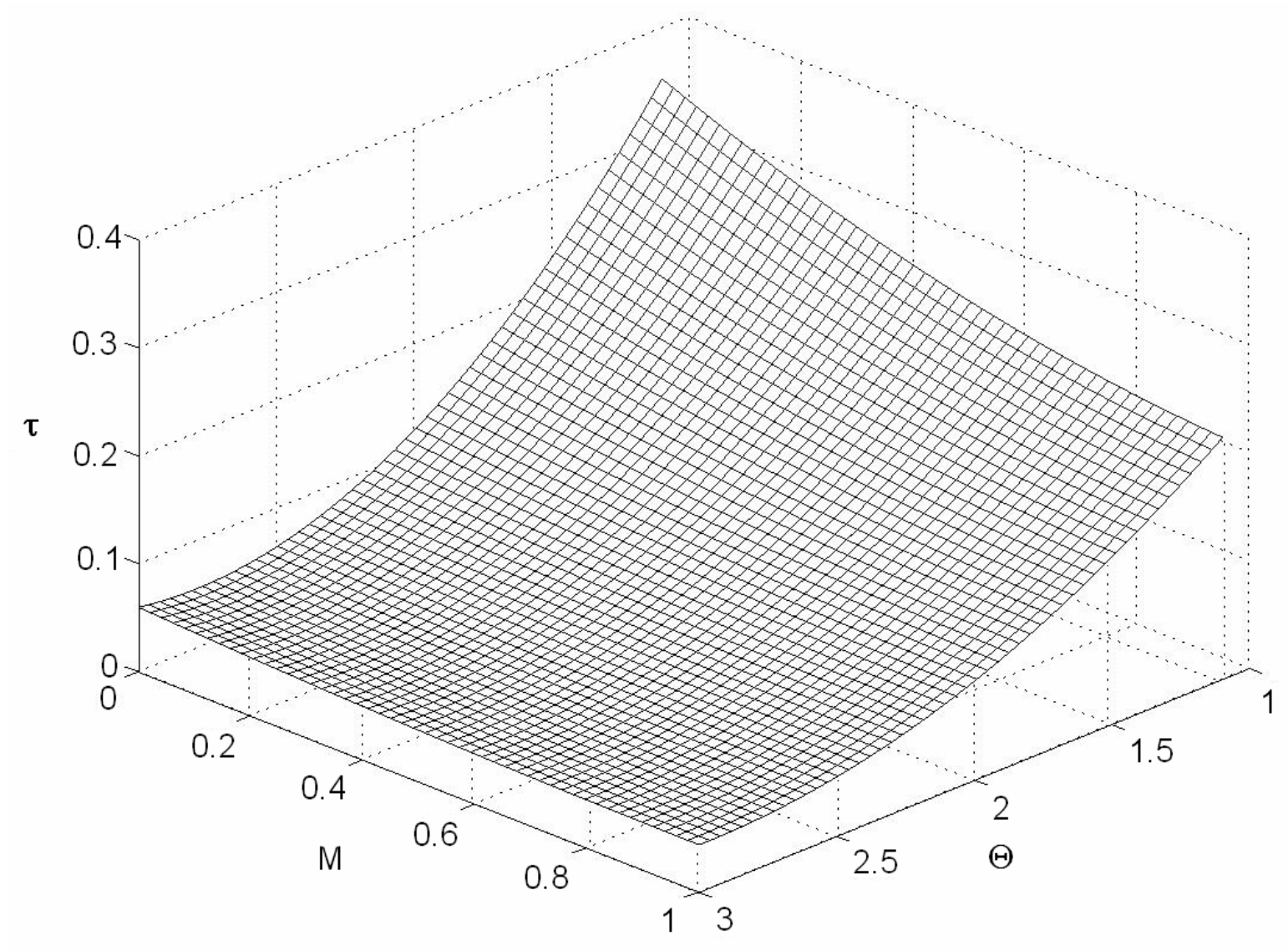

Fig. 13: Influencia de la velocidad del vuelo en valor de $\tau$. 
El sistema de AFH tiene la mayoría de la eficacia si el ángulo $\beta$ de la inyección del chorro es $135^{\circ}$ (fig. 10) relativamente el sentido de flujo principal (en este caso el consumo de aire del chorro transversal es mínimo para crear la región de circulación).

Como muestra fig. 11, el aumento de bloqueo del canal $f$ y de la calefacción relativa $\theta$ en el dispositivo de poscombustión aumenta el valor relativo del tiempo $\tau$. Este resultado es provocado por el aumento de la velocidad local debido a bloqueo del canal, y por aumento correspondiente de la resistencia aerodinámica de los estabilizadores de la llama en concordancia con curva del segundo orden (39). Al mismo tiempo, el modelo utilizado lineal no permite estimar precisamente la influencia del bloqueo en $\tau$ para grandes $f(f>0,3)$, porque la relación entre los parámetros de turboventilador y bypass del aire no es lineal para el consumo elevado del aire.

La influencia del coeficiente $\lambda$ de la velocidad inicial en entrada del dispositivo de poscombustión se demuestra en fig. 12. Un cierto aumento de $\tau$ con el aumento de $\lambda$ ocurre por las razones dadas arriba. Está claro que los valores de $\tau$ son limitados por la línea de evacuación del flujo.

La fig. 13 demuestra que el incremento del número de Mach del vuelo reduce valor de $\tau$, es decir, una ventaja del sistema de AFH disminuye cuando el número de Mach del vuelo aumenta.

Para resumir, se puede concluir que el sistema de AFH se puede recomendar como unidad de la reserva con aumento moderado del empuje para el uso en la operación de emergencia (distancia escasa del despegue, daño de motor, situación de emergencia) o en la operación corta del tiempo (romper la barrera del sonido, alcanzar la altura máxima, del despegue).

\section{CONCLUSIONES}

El modelo matemático lineal del motor de turboventilador con del dispositivo de poscombustión fue desarrollado para analizar una influencia de la sangría del compresor con el bypass del aire para el sistema de AFH en las características de turboventilador.
Un criterio de la evaluación fue propuesto para comparar una eficacia de sistema de los sostenedores mecánicos de la llama y sistema aerodinámico de los sostenedores de la llama.

Una influencia de diversos parámetros (velocidad del vuelo, velocidad de aire en la entrada del dispositivo de poscombustión, relación de la calefacción del dispositivo de poscombustión, ángulo de la inyección de los chorros transversales y factor de bloqueo del canal) en este criterio era analizada.

Los factores que causan el aumento de la pérdida de la presión en el dispositivo de poscombustión sin funcionamiento, aumentan la región donde está preferible el uso del sistema de AFH en comparación con sistema de $\mathrm{MFH}$.

El sistema de AHF puede ser recomendado como unidad de reserva para aumento moderado del empuje para uso en operación de emergencia (escaso distancia para despegue, daño de motor) o en corto tiempo de operación (para romper la barrera de son, para alcanzar la altura máxima, para despegue).

\section{REFERENCIAS}

Alverman, W. y R. Ulken, Aerodynamische Flammenstabilisierung, Experimmentetelle Untersuchungen und Anwendungsmöglichkeiten, Z. Flugwiss, 19(4), 168-178, (1971).

Balas, G. J., Linear, parameter-varying control and its application to a turbofan engine, International Journal of Robust and Nonlinear Control, 12, 763-796, (2002).

Cherkez, V.J., Cálculo dos Turboreatores pelo Método dos Desvios Pequenos (in Russian), Mashinostroenie, Moscou, (1975).

Doel, D. L., TEMPER - A Gas-Path Analysis Tool for Commercial Jet Engines, Journal of Engineering for Gas Turbines and Power, 116, 82-89, (1994)

Gilyazov, M.S., V.A. Kosterin, y T.N. Motylinskaya, Calculation of Aerodynamic Drag of Aerodynamic Flameholders (in Russian), Transactions of Kazan Aircraft Production College, 156, 48-52, (1973). 
Gollahalli, S. R. y D. Pardiwalla, Comparison of the Flame Characteristics of Turbulent Circular and Elliptic Jets in a Crossflow, Journal of Energy Resources Technology, 124(3), 197-203, (2002).

Henrion, D., L. Reberga, J. Bernussou, F. Vary, Linearization and Identification of Aircraft Turbofan Engine Models, Proceedings of $16^{\text {th }}$ IFAC Symposium on Automatic Control in Aerospace, St. Petersburg, Russia, May 1418, (2004).

Khatchatourian, O.A., Combustion de Querosen em Aire, en Condiciones de Estabilizacion Aerodinamica de la Llama, Información Tecnologica, 9(4), 247-254 (1998).

Khatchatourian, O.A., Theoretical and Experimental Study on the Application of the Aerodynamical Flame Stabilizer in Transversal Ring Form Applied to a Turbo Reactor Secondary Combustion Chamber, in Proceedings of $2^{\text {nd }}$ Italian-Latinoamerican Conference on Applied and Industrial Mathematics, ITLA 97, 149, Rome, Italy, January 27-31, (1997).

Khatchatourian, O.A., Experimental Study of the Turbulent Wake Downstream of a Fan Jet, Russian Aeronautics, Allerton Press, USA, 21(3), 72-77 (1978).

Koopman, J., M. Rachner, H. Wiegand y $\mathrm{H}$. Eickhoff, Aerodynamics and Stabilization of Combustion of Hydrogen Jets Injected into Subsonic Airflow. In: AGARD, Hypersonic
Combined Cycle Propulsion, Conference Proceedings, No. 479, 1-16, Madrid, Spain, 28 May - 1 June, (1990).

Li, Z., S. Murugappan, E. Gutmark, y L. Vallet, Numerical Simulation and Experiments of Jets in Cross Flow, 44th AIAA Aerospace Sciences Meeting and Exhibit; Reno, NV, USA, (2006)

Liu, F y W.A. Sirigano, Turbojet and Turbofan Engine Performance Increases Through Turbine Burners, Journal of Propulsion and Power, 17(3), 695-705, (2001).

Raushenbakh B.V., S.A Belyi, y I.V. Bespalov, Physical Principles of the Working Process in Combustion Chambers of Jet Engines, Foreign Technology Division, Wright Patterson Air Force Base, Ohio, (1964).

Shliakhtenko, G.P., Theory of Jet Engines (in Russian), Mashinostroenie, Moscou, (1985).

Simani, S., Patton, R. J., Daley, S. y Pike, A., Identification and Fault Diagnosis of an Industrial Gas Turbine Prototype Model, Proceedings of the $39^{\text {th }}$ IEEE Conference on Decision and Control, Sydney, Australia, December (2000).

Urban, L. A. y Volponi, A. J., Mathematical Methods of Relative Engine Performance Diagnostics, Transactions Journal of Aerospace, Section 1, Vol. 101, Technical Paper No. 922048, (1992). 